\title{
OCORRÊNCIA DE LARVAS PEDIVÉLIGER DE OSTRAS DO GENERO CRASSOSTREA SACCO, 1897 NO SETOR EUHALINO DO COMPLEXO ESTUARINO DE PARANAGUÁ - PR
}

\section{OCCURRENCE OF LARVAE PEDIVELIGER OYSTERS OF THE GENUS CRASSOSTREA SACCO, 1897 IN THE EUHALINE SECTOR OF PARANAGUÁ ESTUARINE COMPLEX-PR}

\author{
Susete Wambier Christo'; Silvio Luiz Ferreira² ${ }^{\text {; Theresinha Monteiro Absher }}$; \\ Augusto Luiz Ferreira Júnior ${ }^{4}$ \\ ${ }^{1}$ Universidade Estadual de Ponta Grossa, Departamento de Biologia Geral, Ponta Grossa - \\ PR; e-mail: swchristo@hotmail.com \\ ${ }^{2}$ Graduando em Oceanografia, Centro de Estudos do Mar/ Universidade Federal do Paraná - PR \\ ${ }^{3}$ Centro de Estudos do Mar/ Universidade Federal do Paraná - PR \\ ${ }^{4}$ Mestrando em Aquicultura, Universidade Federal de Santa Catarina - SC
}

Data de recebimento: 20/10/2013

Data da aprovação: 12/11/2013

\section{RESUMO}

O desenvolvimento larval planctônico e planctotrófico de ostras do gênero Crassostrea é caracterizado por três estágios larvais: larva $\mathrm{D}$, Umbo e Pedivéliger. Nesta última fase as larvas encontram-se próximas ao assentamento, onde ocorre a fixação e metamorfose. Neste trabalho estudou-se a ocorrência de larvas de ostras do gênero Crassostrea no Complexo Estuarino de Paranaguá (CEP) e sua correlação com parâmetros ambientais como salinidade, temperatura e turbidez da água do mar. Realizaram-se coletas de plâncton através de arrasto com duas repetições, no período de inverno/2011 e verão/2012, na Ilha do Mel, Canal da Galheta (Ponto I), e na desembocadura da Gamboa do Rio Maciel (Ponto II). O material biológico foi analisado em laboratório e as larvas quantificadas por amostragem total. O resultado indicou um número maior de larvas nos meses de verão, sugerindo que os processos de maturação gonadal são desencadeados pela elevação da temperatura da água do mar. O maior número de larvas verificado na desembocadura do Rio Maciel (Ponto II) pode ser devido à proximidade a uma região de cultivo.

Palavras-chave: Plâncton. Estuário. Desenvolvimento larval. Crassostrea.

\begin{abstract}
The planktonic and planktotrophic larval development of oysters of Crassostrea genus is characterized by three larval stages: larva D, Umbo and Pediveliger. In this last phase the larvae are at the end of the larval cycle and near metamorphosis. In this work the occurrence of oyster's larvae of Crassostrea genus was studied in the Paranaguá Estuarine Complex (PEC) and the correlation with environmental parameters as salinity, temperature and seawater turbidity. Plankton samples were collected in two replicates in winter/2011 and summer/2012 in two sampling stations: Point I - Galheta Channel, Ilha do Mel and Point II - Maciel river outlet. In the laboratory, the samples were analyzed, and the total number of larvae counted. Results indicated a higher number of larvae in the summer months suggesting that the processes of gonadal maturation are linked to
\end{abstract}


higher seawater temperature. The highest number of larvae verified in Maciel's river outlet (Ponto II) may be due to the proximity to a cultivation area.

Keywords: Plankton. Estuaries. Larval development. Crassostrea.

\section{Introdução}

As ostras ocupam um lugar destacado nas comunidades estuarinas, formando bancos na região entremarés e no infralitoral, tanto em substratos rochosos como nas raízes do mangue. São moluscos bivalves, sésseis quando adultos, pertencentes à família Ostreidae. No litoral do Paraná ocorre o gênero Crassostrea (SACCO, 1897), caracterizado por apresentar grande plasticidade na morfologia da concha na dependência do substrato onde estão fixados os indivíduos (ABSHER, 1989).

As ostras do gênero Crassostrea possuem ausência de dimorfismo sexual, com fecundação externa e desenvolvimento larval planctotrófico (STRATHMANN, 1992). Ao final do período larval, quando se encontram próximas ao assentamento, tendem a concentrar-se junto ao fundo, onde ocorre a fixação e a metamorfose em substrato duro (ABSHER, 1989; BAKER, 2003; FINELLI; WETHEY, 2003).

O desenvolvimento larval das ostras é caracterizado por três estágios: larva D - comum a todos os bivalves; larva umbo - em Ostreidae apresenta assimetria do umbo em relação ao plano anteroposterior da concha; e pedivéliger - corresponde à última fase do ciclo larval e é caracterizado por larvas com altura acima de $300 \mu \mathrm{m}$. (LE PENNEC, 1980; ABSHER et al., 2000; CHRISTO et al., 2010). Neste estágio, as larvas nadam ativamente pelo uso do velum (DEKSHENIEKS et al.,1996), sendo as espécies diferenciadas através do número de dentes da prodissoconcha (CHRISTO et al., 2010).

A duração do período larval no plâncton é determinada principalmente pela temperatura da água e também pela disponibilidade de alimento (ABSHER, 1989; CHRISTO, 2006). Porém, fatores como salinidade e turbidez podem inibir o crescimento, causar mortalidade das larvas, retardar o crescimento ou interferir na dispersão das mesmas na natureza (LIANG et al., 2000; REN et al., 2003).

Em estuários, apesar do transporte de organismos para regiões oceânicas pela água da maré vazante com maior velocidade e volume de água, ainda se observa uma alta densidade de larvas que permanecem dentro dos estuários. Este processo sugere uma interação entre o comportamento de natação das larvas e as características de circulação do ambiente (SILVA; ABSHER, 1996; BOEHS; ABSHER, 1997). A retenção de larvas pelágicas estuarinas também é influenciada pelo transporte ativo e passivo das mesmas (MANN et al., 1991; SILVA; ABSHER, 1996; BOEHS; ABSHER, 1997). Alguns estudos sugerem que a larva pode manter sua posição na coluna d água independentemente de fatores ambientais (WOOD; HARGES, 1971; HIDU; HASKIN, 1978).

À luz dessas considerações, este trabalho teve como objetivo verificar a ocorrência de larvas de ostras em estágio pedivéliger em dois pontos do setor euhalino do CEP e correlacionar os dados biológicos com parâmetros ambientais. como temperatura da água do mar, salinidade e transparência da água.

\section{Material e Métodos}

O litoral do Paraná, localizado no sul do Brasil, estende-se desde a Vila de Ararapira, ao norte $\left(25^{\circ} 12^{\prime}\right.$ $44^{\prime \prime} \mathrm{S} ; 48^{\circ} 01^{\prime} 15^{\prime}$ W), até a barra do Rio Saí-Guaçu, ao sul ( $25^{\circ} 58^{\prime} 38^{\prime \prime} \mathrm{S}$; $\left.48^{\circ} 35^{\prime} 26^{\prime \prime} \mathrm{W}\right)$, e possui dois estuários: o Complexo Estuarino de Paranaguá (CEP) e a Baía de Guaratuba. Segundo Noernberg et al. (2006), o CEP é dividido em cinco setores, conforme características morfológicas, hidrológicas e físicas (Figura 01). As coletas foram realizadas no período de inverno/2011 e verão/2012, em dois pontos no setor euhalino do estuário: o primeiro, próximo a Ilha do Mel, no Canal da Galheta (Ponto I); e o segundo, na desembocadura Gamboa do Rio Maciel (Ponto II), próximo à Ilha Rasa da Cotinga, denominados de Ponto I e Ponto II, respectivamente (Figura 01).

Em cada ponto foram efetuados dois arrastos consecutivos de fundo com duração de 1 minuto cada a uma velocidade aproximada de 2 nós, utilizando-se uma rede cônica de plâncton, com malha de 225 
$\mu \mathrm{m}$ e $30 \mathrm{~cm}$ de diâmetro de abertura. As amostras de plâncton retidas foram fixadas em formol a $4 \%$, previamente neutralizado com tetraborato de sódio, e em seguida preservadas em álcool 70\%. Posteriormente, foram transportadas até o laboratório, onde as larvas de ostras em estágio pedivéliger foram separadas e contadas em microscópio estereoscópico por amostragem total. Os resultados quantitativos referentes ao número de larvas foram transformados em densidade de larvas pedivéliger $/ \mathrm{m}^{3}$.

Figura 01 - Localização dos pontos de amostragens do Complexo Estuarino de Paranágua, Paraná, Brasil. O: Ponto I (Ilha do Mel - Canal da Galheta); $\square$ : Ponto II (Gamboa do Rio Maciel); $\square$ : Área de cultivo suspenso de ostras nativas. 1- Baía de Mistura; 2- Baía de Paranaguá; 3- Baía de Antonina; 4- Baía das Laranjeiras; 5- Baía dos Pinheiros. (NOERNBERG et al,. 2006).

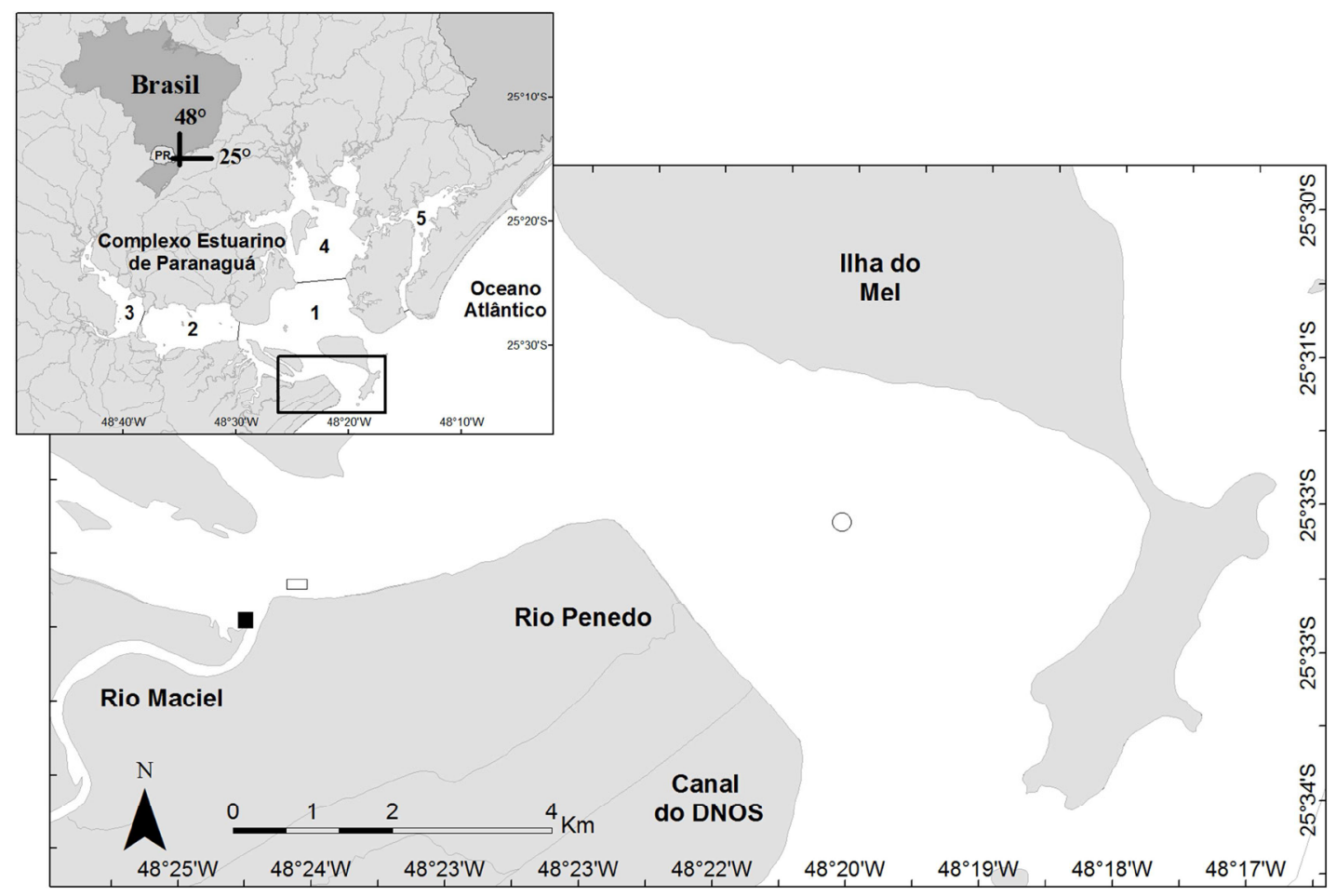

Paralelamente às coletas foram monitorados os seguintes parâmetros ambientais: temperatura da água do mar, medida com termômetro de mercúrio (precisão de $0,1^{\circ} \mathrm{C}$ ); salinidade, com o uso de um refratômetro portátil; e a transparência da água, estimada através do Disco de Secchi (profundidade de extinção). A altura da maré foi estimada através da tábua de previsão de preamares e baixa-mares da Diretoria de Hidrografia e Navegação (DHN), Marinha do Brasil, para o Porto de Paranaguá.

Os dados de densidade larval foram transformados (raiz quadrada) e submetidos a Anova bi-fatorial (local e estação).

\section{Resultados}

Quanto à distribuição das larvas, as análises mostraram uma densidade média de 22,00 $(\mathrm{DP} \pm 27,43)$ larvas $/ \mathrm{m}^{3}$ no inverno e $9,00(\mathrm{DP} \pm 4,38)$ larvas $/ \mathrm{m}^{3}$ no verão, para o Ponto I; enquanto que o Ponto II apresentou uma densidade média de $23,80(\mathrm{DP} \pm 29,26)$ lar$\operatorname{vas} / \mathrm{m}^{3}$ no inverno e $106,80(\mathrm{DP} \pm 83,93)$ larvas $/ \mathrm{m}^{3}$ no verão, durante o período estudado. A análise mostrou que não houve diferença significativa entre as densidades de larvas pedivéliger ( $\mathrm{p}>0,05)$ nos Pontos I e II nos meses de inverno (junho a agosto/2011) (Figura 02). No entanto, nos meses de verão (dezembro/2011 a fevereiro/2012), as diferenças entre as densidades de larvas observadas entre os dois pontos foram significativas $(\mathrm{F}=7,11 ; \mathrm{p}=0,02)$ (Figura 02$)$. Os resultados das contagens indicaram a ocorrência de larvas de ostras no plâncton durante o período amostrado, com maior abundância no verão, sendo que o Ponto II apresentou uma maior densidade de larvas pedivéliger $/ \mathrm{m}^{3}$ em dezembro/2011 (Figura 03). 
Figura 02 - Densidade de larvas pedivéliger nos Pontos I (Ilha do Mel) e Ponto II (Gamboa do Rio Maciel), durante o período amostrado.

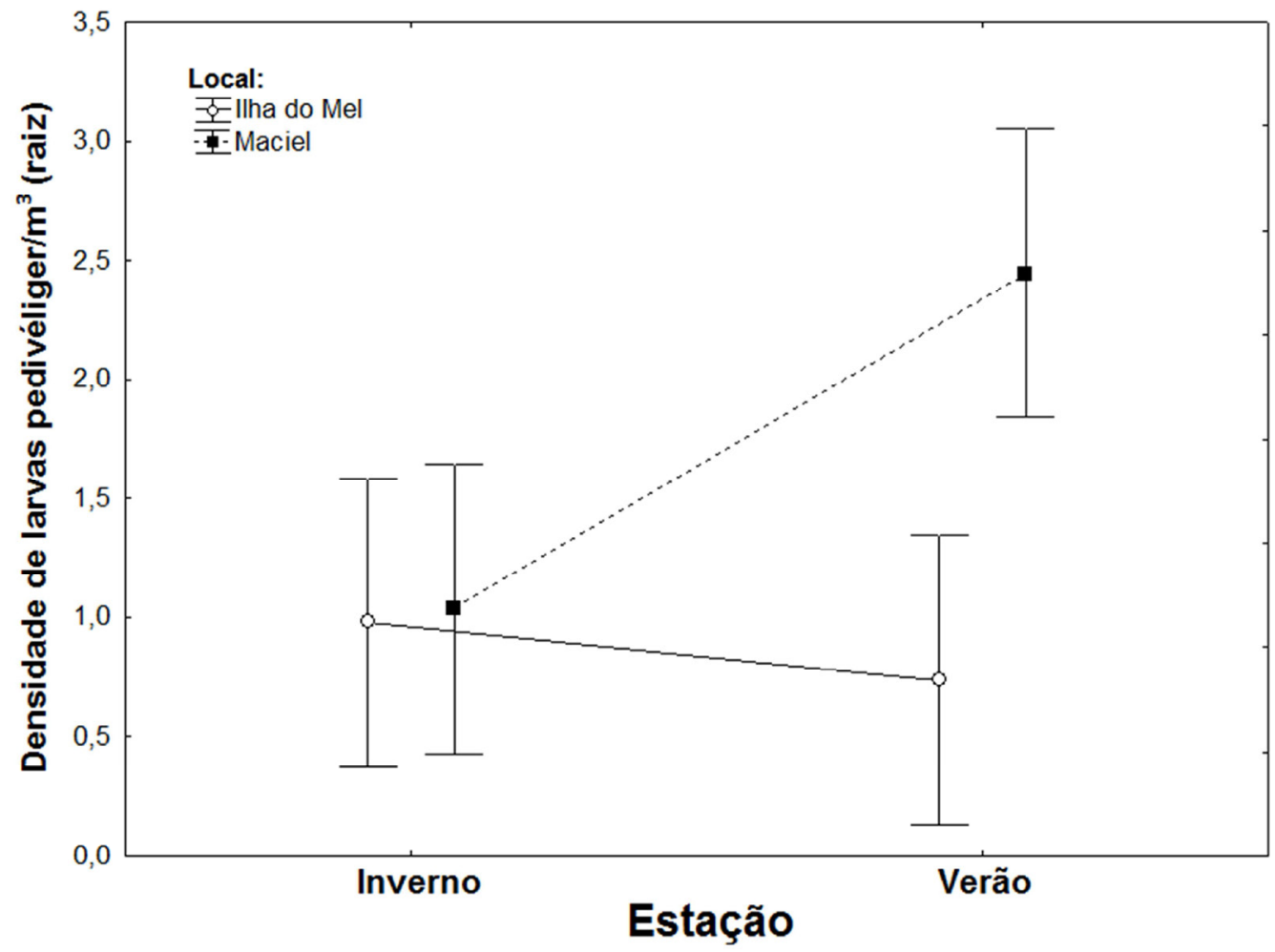

Figura 03 - Densidade de larvas pedivéliger no período inverno/verão nos Pontos I (Ilha do Mel) e Ponto II (Gamboa do Rio Maciel).

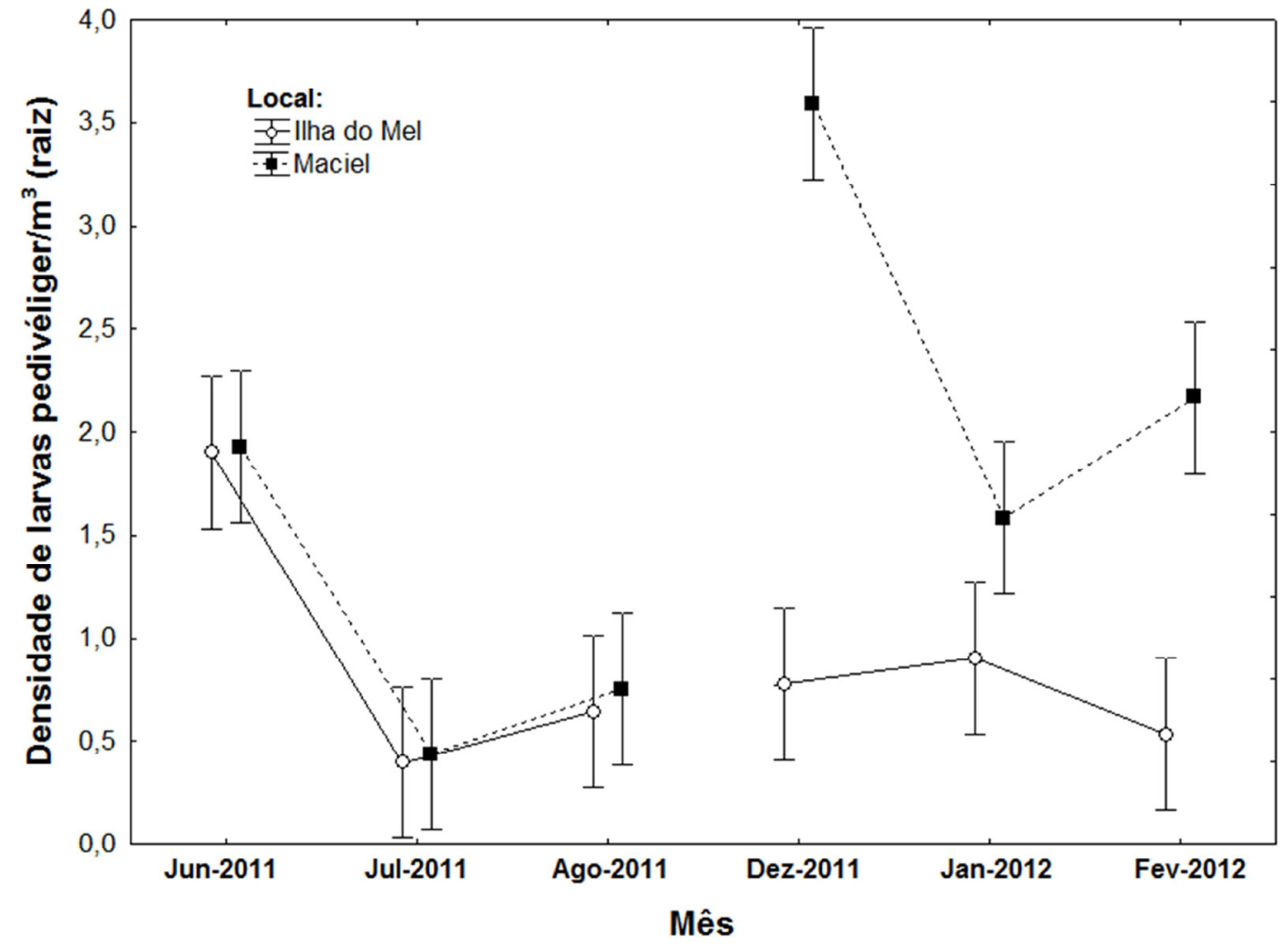


A temperatura da água do mar apresentou valores que oscilaram entre $17^{\circ} \mathrm{C}$ e $29^{\circ} \mathrm{C}$, com média de $17,4^{\circ} \mathrm{C}$ no inverno e $27,8^{\circ} \mathrm{C}$ no verão, nos Pontos I e II, respectivamente. A temperatura mais alta $\left(29^{\circ} \mathrm{C}\right)$ foi registrada no período de janeiro/2012 em ambos os pontos estudados. A salinidade variou entre $27 \mathrm{e}$ 32 , com média de 30,5 para o Ponto I e 29,5 para o Ponto II. O menor valor registrado (27) corresponde à área da desembocadura da Gamboa do Rio Maciel. Quanto à profundidade de extinção do Disco de Secchi, a média foi de 2,00 m no Ponto I e $0,75 \mathrm{~m}$ para o Ponto II, sendo o maior valor de transparência da água verificado no Ponto I (3,00 m), correspondente à área de entrada do $\mathrm{CEP}$, e o menor valor registrado $(0,60 \mathrm{~m})$ no Ponto II.

\section{Discussão}

O ciclo reprodutivo da ostra é constituído por uma série de etapas que envolvem desde a gametogênese até a fixação e metamorfose, sendo influenciadas por fatores endógenos e exógenos. Os fatores exógenos que têm maior influência sobre a maturação gonadal e duração do ciclo larval são a temperatura e a disponibilidade de alimento no ambiente. (GALVÃO et al., 2000; KREEGER et al., 2003; REN et al., 2003; ORBAN et al., 2004).

Os resultados obtidos no presente trabalho sugerem que as ostras do Complexo Estuarino de Paranaguá possuem um padrão de reprodução continuo devido à presença de larvas no plâncton em períodos de inverno e verão, porém, com maior influência da temperatura, pois a alta densidade de larvas coincide com períodos de elevação da temperatura e maturação gonadal de ostras adultas (ABSHER, 1989; CHRISTO: ABSHER, 2004). Silva e Absher (1996) constataram que a presença de larvas de ostras no Complexo Estuarino de Paranaguá ocorre o ano todo, com intensificação nos períodos mais quentes em consequência da alta temperatura da água e picos nos períodos mais expressivos de desova no outono, primavera e verão.

Estudos com larvas de moluscos C. rhizophorae e $C$. brasiliana indicam que temperatura, salinidade e alimento disponível podem interferir na duração e distribuição de larvas no plâncton (QUAYLE, 1988; LIANG et al., 2000; SILVA; ABSHER, 1996; BOEHS: ABSHER, 1997; KERN et al., 2012). Boehs e Absher (1997), em estudos com larvas de ostras na
Baía de Paranaguá, sugerem que indivíduos próximos ao assentamento possuem algum mecanismo de deslocamento ativo que os auxilia a permanecer no interior do estuário.

Outros fatores ambientais, que podem determinar especialmente o transporte horizontal das larvas, são: ondas, velocidades das correntes e marés. Marone et al. (2005) identificam, no setor que engloba o Canal da Galheta, média da velocidade de corrente das massas d'água de $0,30 \mathrm{~m} / \mathrm{s}$ para o período chuvoso e de $0,34 \mathrm{~m} / \mathrm{s}$ para o período seco do ano. Já a região da Gamboa do Rio Maciel é caracterizada por possuir os menores valores de densidade de drenagem $(0,67$ rios $/ \mathrm{km}^{2}$ ) e gradiente hidráulico (elevação/extensão do rio) do CEP (NOENBERG et al., 2006).

Kern et al. (2012) identificaram uma distribuição de larvas de moluscos no CEP, com maior predominância na região euhalina, caracterizada como setor de mistura. Estes mesmos autores descrevem o maior número de larvas de ostras próximo a desembocadura da Gamboa do Rio Maciel.

No Ponto I observa-se uma menor densidade de larvas no último estágio do desenvolvimento (pedivéliger), isto pode estar associado à ausência de sistemas fluviais e características oceanográficas com maior dinâmica nesta região Boehs e Absher (1997), em um ponto mais externo do Canal da Galheta, identificaram valores semelhante aos resultados obtidos no presente trabalho.

Larvas no último estágio do desenvolvimento (pedivéliger) possuem uma capacidade de natação e/ ou deslocamento aumentado devido ao seu tamanho. Este fato pode indicar a alta densidade de larvas pedivéliger próximas ao assentamento, observadas especialmente no Ponto II no período de verão. Nesta região, próxima à desembocadura da Gamboa do Rio Maciel, foi implantado um parque aquícola de cultivo suspenso (long-lines) de ostras nativas. A elevação da temperatura da água do mar (verão) associada à proximidade do parque aquícola pode justificar a ocorrência de quantidades maiores de larvas neste local, onde há um retorno das mesmas aos locais de fixação. Além do aporte de larvas oriundas do parque aquícola nesta região, também há a contribuição dos estoques naturais localizados na Gamboa do Rio Maciel. Comportamento semelhante foi observado por Boehs e Absher (1997) para larvas de ostras nesta região da Baía de Paranaguá em estudos efetuados 
anteriormente à implantação do parque aquícola. Este estudo também corrobora o fato de larvas de ostras do gênero Crassostrea serem consideradas estuarinas, eurihalinas e adaptadas aos ambientes de turbidez elevada (QUAYLE, 1988, GALVÃO et al., 2000). Estudos efetuados em laboratório indicaram maiores sobrevivências de sementes de ostras nativas em águas com salinidade variando de 15 a 25 (GUIMARÃES et. al., 2008).

\section{Conclusão}

Os resultados obtidos neste trabalho indicam a ocorrência de larvas de ostras, com maior intensidade em períodos de elevação de temperatura da água do mar, preferencialmente em área mais abrigadas e/ ou próximas aos sistemas de cultivo implantados na região.

\section{Referências}

ABSHER, T. M. Populações naturais de ostras do gênero Crassostrea do litoral do Paraná Desenvolvimento larval, recrutamento e crescimento. Tese de doutorado. Universidade de São Paulo, Instituto Oceanográfico, p.185, 1989.

.; VERGARA, E. M. ; CHRISTO, S. W. Growth and allometry of the larval shell of the brazilian oyster Crassostrea brasiliana (Lamark, 1819) (BIVALVIA:OSTREIDAE). OPHELIA. v.53, n.2 , p.105-112, 2000.

BAKER, P. Two species of oyster larvae show different depth distrubuitions in a shallow, well-mixed estuary. J. Shellfish Res., v.3, n.3, p. 733-736, 2003.

BOEHS, G. ; ABSHER, T. M. Distribuição de larvas de ostras do gênero Crassostrea SACCO, 1897. (OSTREOIDA:OSTREIDAE) na Baía de Paranaguá, Paraná. Arq. Biol. Tecnol. v.40, n.1, p.39-45, 1997.

CHRISTO S. W.; ABSHER T. M. Reproductive period of Crassostrea rhizophorae (GUILDING, 1828) and Crassostrea brasiliana (Lamark, 1819) (Bivalvia: ostreidae) in Guaratuba bay, Paraná, Brazil. Journal of Coastal Research, SI 39, p.1215 - 1218. Itajaí, SC, Brazil, 2004.

Biologia reprodutiva e ecologia de ostras do gênero Crassostrea Sacco, 1897 na Baía de Guaratuba (Paraná - Brasil): um subsídio ao cultivo. Tese de doutorado, Universidade Federal do Paraná, Departamento de Zoologia, p.137, 2006.

; ABSHER,T. M. ; BOEHS, G. Morphology of the larval shell of three oyster species of the genus
Crassostrea Sacco, 1897 (Bivalvia: Ostreidae). Braz. J. Biol., v.70, n.3, p. 645-650, 2010.

DEKSHENIEKS, M. M.; HOFMANN, E. E.; KLINCK, J. M.; POEWLL, E. N. Odeling the vertical distribuition of oyster larvae in response to environmental conditions. Mar. Ecol. Prog., v.136, n.1-3, p. 97-110, 1996.

FINELLI, C. M.; WETHEY, D. S. Behavior of oyster (Crassostrea virginica) larvae in flume boundary layer flows. Mar. Biol., v.143, n.4, p.703-711, 2003.

GALVÃO, M. S. N.; PEREIRA, O. M.; MACHADO, I. C. ; HENRIQUE, M. B. Aspectos reprodutivos da ostra Crassostrea brasiliana de manguezais do estuário de Cananéia, SP $\left(25^{\circ} \mathrm{S} ; 48^{\circ} \mathrm{W}\right)$. B. Inst. Pesca, v. 26, n. 2, p.147-162, 2000.

HIDU, H.; HASKIN, H. H. Swimming speeds of oyster larvae Crassostrea virginica in different salinities and temperatures. Estuaries, v.1, n.4, p.252-255,1978.

KREEGER, D.; THOMAS, R.; HERTLER, H. ; RAKSANY, D. Spatial and temporal variation in oyster fitness in San Antonio Bay, Texas, 1998-2002. J. Shellfish Res. v.22 n.1, p.338-339, 2003.

LE PENNEC, M. The larval and post-larval hinge of some families of bivalves molluscs. J. Mar. Biol. Ass., v.60, p.601-617, 1980.

LIANG, X.; FANG, J.; TANG, Q.; JIANG, W.; PENG, S.; JI, Y. Studies on prevention of the fouding oyster larvae from attaching to cultured bay scallop (Argopecten irradians Lamark) in Jincheng cultivation area of Laizhou Bay. Mar Fish. Res., v.21 , n.1, p. $27-$ 30, 2000.

MANN, R.; CAMPOS, B. M.; LUCKENBACH, M. W. Swimming rate and responses of larvae three mactrid bivalves to salinity discontinuities. Mar. Ecol. Prog. Ser., v. 68 p. 257-269, 1991.

NOERNBERG, M. A.; LAUTERT, L. F. C.; ARAÚJO, A. D.; MARONE, E.; ANGELOTTI, R.; NETTO JR., J. P. B. ; KRUG, L. A. Remote Sensing and GIS Integration for Modeling the Paranaguá Estuarine Complex -Brazil. Journal of Coastal Research. v.39, p.1627-1631, 2006.

ORBAN, E.; LENA, G.; MASCI, M.; NEVIGATO, T.; CASINI, I.; CAPROLI, R.; GAMBELLI, L. ; PELLIZATO, M. Growth, nutricional quality and safety of oysters (Crassostrea gigas) cultured in the Lagoon of Vence (Italy). J. Sci. Food. Agric. v.84 n. 14, p. 1929-1938, 2004. 
QUAYLE, D.R. Pacific oyster culture in Britsh Columbia. Bull. Fish. Aquat. Sci., v. 218 p. 1-241, 1988.

REN, J. S.; ROSS, A. H.; SCHIEL, D. R. Functional descriptions of feeding and energetics of the Pacific oyster Crassostrea gigas in New Zealand. Mar. Ecol. Prog. Ser. v. 208, p. 119-130, 2003.

SILVA, G. B.; ABSHER, T. M. Variação temporal de larvas de ostras do gênero Crassostrea SACCO, 1897 (Ostreoida:Ostreidae) na Baía de Paranaguá, Paraná. Arq. Biol. Tecnol. v. 39, n.4, p. 903-910, 1996.

STRATHAMANN, M. N. Reproduction and development of marine invertebrates of the Northen Pacific Coast. University of Washinton Press, v. 2, p. 670, 1992.

WOOD, L.; HARGES, JR, W. J. Transport of bivalve larvae in a tidal estuary. Proc. Eur. Mar. Biol. Symp., v. 4, p. 29-44, 1971. 